\section{Cureus}

Received 05/05/2015

Review began 05/08/2015

Review ended 05/18/2015

Published 05/27/2015

\section{(c) Copyright 2015}

Ahmed et al. This is an open access article distributed under the terms of the Creative Commons Attribution License CC-BY 3.0., which permits unrestricted use, distribution, and reproduction in any medium, provided the original author and source are credited.

\title{
Perceptions and Preferences of Patients with Terminal Lung Cancer and Family Caregivers about DNR
}

Naseer Ahmed ${ }^{1}$, Michelle Lobchuk ${ }^{2}$, William M. Hunter ${ }^{1}$, Pam Johnston ${ }^{3}$, Zoann Nugent ${ }^{4}$, Ankur Sharma ${ }^{5}$, Shahida Ahmed ${ }^{1}$, Jeff Sisler 6

1. Radiation Oncology, CancerCare Manitoba, University of Manitoba 2. Faculty of Nursing, Cancer Care Manitoba, University of Manitoba, Canada 3. Department of Nursing, Cancer Care Manitoba, University of Manitoba, Canada 4. Department of Epidemiology and Cancer Registry, Cancer Care Manitoba, University of Manitoba, Canada 5. Cancer Care Manitoba, Cancer Care Manitoba, University of Manitoba, Canada 6. Family Medicine, Cancer Care Manitoba, University of Manitoba, Canada

$\square$ Corresponding author: Naseer Ahmed, nahmed2@cancercare.mb.ca

Disclosures can be found in Additional Information at the end of the article

\section{Abstract}

Background: Patients with terminal lung cancer and their families are challenged and stressed with the end of life discussions. Do Not Resuscitate (DNR) orders are a critical part of such discussions.

Objective: To understand the perceptions and preferences of patients with terminal lung cancer and their family caregivers around DNR discussions. .

Methods: Our quantitative component consisted of a pen-and-paper questionnaire that was followed by a 'think aloud' process to capture perceptions of participants in response to questionnaire items. Qualitative methods included content analysis and constant comparison techniques to identify, code, and categorize primary themes arising from 'think aloud' responses.

Results: In this pilot study, 10 patients with advanced stage lung cancer and nine family caregivers were enrolled from one tertiary cancer care centre. Three major themes and several sub-themes were identified reflecting participants' psychosocial environment, emotional responses to DNR discussions, and suggestions to improve DNR discussions. Most of the time, both patients and caregivers perceived a supportive environment within their family unit. Some patients were uncertain about their disease extent but most had entertained thoughts about prognosis and DNR status prior to having a discussion with their physician. A range of situations stimulated the DNR discussion. Most patients were uncertain about identifying the most appropriate health care provider (HCP) for DNR discussion. While participants found DNR discussions distressing, patients maintained hope in the face of accepting a terminal diagnosis. There were mixed feelings about the reversibility of a DNR decision and concerns about the care of the patients after being stated as DNR. Participants desired their HCP to be emotionally sensitive, knowledgeable, respectful, and straightforward.

Conclusions: Most participants were open about their experiences with psychosocial supports and emotional reactions and made suggestions to HCP to improve DNR discussions. Further examination in larger longitudinal studies is required to validate the observations in the current study. 
Categories: Oncology

Keywords: dnr, end of life care, patients and care givers, terminal lung cancer

\section{Introduction}

Patients diagnosed with terminal lung cancer have a rapidly declining performance status and are frail and emotionally vulnerable. Both patients and their families are confronted with the difficult end of life (EOL) discussions, and decisions sometimes need to be made within days after receiving a terminal diagnosis. Do Not Resuscitate (DNR) orders are a significant part of EOL discussions [1]. We recently published a report describing perceptions and preferences of patients, their family caregivers (CGs), and their health care providers (HCP), with respect to their understanding of and the appropriate time to discuss DNR [2]. In the current article, the investigators report study findings from their cross-sectional exploration of cognitive and emotional perceptions and preferences of patients and their CGs when engaged in DNR discussions.

\section{Materials And Methods}

In this pilot study, 10 patients and nine CGs who met the eligibility criteria were recruited from Cancer Care Manitoba (CCMB) in Canada where tertiary and multidisciplinary care are provided to approximately 800 newly diagnosed lung cancer patients per year. Once considered incurable, patients are given the option to enroll into a palliative care program. Patients must have a DNR status to be eligible for this program. Patients with a pathological diagnosis of either non-small cell or small cell lung cancer and incurable disease, who did not exhibit confusion, and who were not to receive further curative therapy, were eligible. Additional eligibility criteria included a discussion with the HCP around DNR, and having a CG who was willing to participate in the study. A CG was identified by the patient as the individual who assisted most with his or her care and was most involved with the patient in the DNR status decision-making was eligible to participate in the study.

Quantitative and qualitative methods were used to capture perceptions of study participants. A dedicated research nurse was trained on the interview technique before she conducted interviews of the participants.

A written informed consent was obtained from the participants. Prior to commencing the study's protocols, the investigators obtained ethics approval from the Research Ethics Board University of Manitoba (approval \#H2011: 227).

\section{Quantitative Component}

Study participants completed respective pen-and-paper versions of an investigator-developed questionnaire for patients and CGs. In these tools, we collected socio-demographic and medical information, which were verified with the patient's medical record with permission from the patient in the written informed consent.

\section{Qualitative Component}

We employed a 'think aloud' process where participants were prompted to immediately verbalize their thoughts when responding to items in the investigator-developed questionnaire on DNR status and DNR discussions [2]. In response to our prompts, we were able to glean an understanding of their level of understanding, personal wishes, triggers, timing, appropriate setting, emotional experiences, appropriate health care provider, and extent of family dialogue on DNR decisions. Interviews were audio-recorded and transcribed for interpretation and analyses of the key themes. A more detailed description of the interview process is captured in 


\section{Cureus}

another article [2].

\section{Data Analyses}

Demographic and medical characteristics, as well as subgroup responses to questionnaire items, were captured by descriptive statistics (Tables 1-2). 


\section{Cureus}

Characteristics

Median age

Gender

M

F

Religious affiliation

Affiliated to some religion

9

Not affiliated to any religion

1

Histological diagnosis of lung cancer

Non-small cell

9

Small cell

1

DNR status

DNR

9

No DNR

Brain metastasis

Present

Absent

7

Whole brain radiation prior to interview

Received

2

Not received

8

Location of the interview

Outpatient clinic

8

In hospital

2

CGs accompanying the patient during study interview

Yes

8

No

2

\section{TABLE 1: Patients $(n=10)$ Characteristics}




\section{Cureus}

Median age

Gender

M

F

Relationship

Spouse

Parents

Sibling

Friend

Religion

Anglican

Lutheran

Greek Catholic

United Church

None

Culture/ethnicity

Scottish, Italian

French, Quebec

1

Icelandic Irish

1

Irish English

1

Scottish, German

2

Ukrainian

2

Ukrainian polish dutch

\section{Education}

High school or less

University

Income

40-80,000K

$<20 K$

Occupational status 


\section{Cureus}

Working part-time or full-time

Retired
Frequency of contact with patient

Living with patient

Visiting weekly
7

2

\section{TABLE 2: Caregivers (CGs) $(n=9)$ Characteristics}

\section{Qualitative Component}

The trained medical transcriptionist transcribed audiotapes of participants' 'think aloud' responses to questionnaire items and semi-structured interview questions. Content analysis and constant comparison techniques were employed by the investigators to identify, code, and categorize primary themes in the transcribed data [3].

\section{Themes and Coding}

Themes and sub-themes from transcribed data obtained from both groups of participants were extracted by three members of the investigative team (NA, WH, and ML). To address qualitative rigor, a detailed audit trail was kept to ensure accurate research procedures were followed. Each investigative team member who independently reviewed the transcribed data kept memos to record theoretical and interpretive insights. The investigative team discussed prominent themes and engaged in a constant comparison of emergent categories. The investigative team met until we achieved consensus on prominent themes and sub-themes. Themes and subthemes were further refined and reflected in a coding template.

\section{Results}

\section{Participant characteristics}

Study participants consisted of 10 patients with advanced stage lung cancer and nine CGs from the lung cancer clinic. One CG could not be interviewed due to logistic reasons and was excluded from analysis. The majority of patients were: male, a median age of 67.5 years of age, had some religious affiliation, a pathologically confirmed diagnosis of non-small cell lung cancer, an identified DNR status, no brain metastases, and had not received whole brain radiation prior to being interviewed (Table 1). Most caregivers were: female, retired, a median of 67.5 years of age, the spouse who lived with the patient, affiliated with the United Church, varied in ethnic background, had reported having less than or high school education, and earned either between $\$ 40,000$ to 80,000 , or less than $\$ 20,000$ in annual income (Table 2). Most patients and their CGs were interviewed in the outpatient clinic (Table 1).

Three major themes, along with several sub-themes, were identified as central to DNR discussions: patients' and CGs' psychosocial environment, patients' and CGs' emotional responses while engaged in DNR discussions, and suggestions to improve DNR discussions.

\section{Psychosocial environment}




\section{Cureus}

The first main theme of 'Psychosocial environment' portrays reflections and insights of patients and CGs about their mutual interactions in the context of impending death. Five sub-themes describe patients' and CGs' perceptions about their relationships and supports, self-awareness, a previously thought through DNR decision, triggers of the DNR discussion, and the involvement of others in DNR discussions.

Sub-theme: Relationships and Supports

This sub-theme reflected a sense of support perceived by patients as coming from mainly spousal caregivers, siblings, and/or friends of the patients who lived with them. Most of the time, both patients and CGs reported having a supportive environment within their family unit (Table 3). When presenting for the scheduled interview, it was noted that most patients were accompanied by their CGs (Table 1).

\begin{tabular}{|l|l|l|}
\hline & CGs & Patients \\
\hline Always & 6 & 6 \\
\hline Frequently & 2 & 2 \\
\hline Sometimes & 1 & 1 \\
\hline Rarely & 0 & 1 \\
\hline
\end{tabular}

TABLE 3: Psychosocial support perceived by the patients $(n=10)$ and CGs $(n=9)$

Sub-theme: Self-awareness

This sub-theme reflected the degree of understanding of the extent of the disease and prognosis by most participants. A small number of patients expressed uncertainty about the terminal nature of their disease (Table 4). In spite of being told by the physician about their disease stage, some patients seemed to be uncertain about their poor prognosis. As one patient stated "I don't know where I'm at? I mean they told me I have Stage IV". Although some patients appeared uncertain, at the same time they also seemed aware that their disease is terminal. 


\section{Cureus}

Perception

“Don’t know/incurable"

"Incurable"

“Stage 4” 1

"Not early stage, spread to bone, it's aggressive, it could take off like any time." 1

"Not sure - at first they thought it was stage 4, then they thought stage 3. Just about the end of the line there."

"One Dr. told me that I have terminal cancer, 2-3 months, but that Dr. wasn't sure. My oncologist told me 6 months."

"Don't know."

"Don't know, but I knew it was bad."

"Not sure."

TABLE 4: Patients awareness about the extent of their disease and prognosis

Sub-theme: A Previously Thought Through DNR Decision

In this sub-theme, patients and/or family members have entertained thoughts about prognosis and DNR status before a formal DNR discussion with their physician. Nine of 10 patients had a predetermined DNR status and seven of 10 patients had previously considered DNR prior to discussing the topic with a health care provider. One caregiver stated: "It's something I guess you [patient] thought ... I thought about it. ..." that was followed by the patient's response, " Yeah, I don't want to die at home...". That appeared to be an important if not influential aspect in this individual's decision-making about his or her DNR status. Although many patients and CGs had engaged in thought processes about DNR prior to meeting with their HCP, only five of 10 patients had previously signed a document related to their DNR status.

\section{Sub-theme: Triggers of DNR Discussion}

This sub-theme depicts triggers that prompted DNR discussions with the HCP and between patients and their CGs. Table 5 captures a range of situations that stimulated DNR discussions, including admission to hospital, a family member who wanted to talk about DNR, the HCP initiated the conversation, the results of pathology or scans, and brain metastasis. When prompted for further triggers that initiated DNR discussions, most patients described a "situation of emergent care". One patient stated: "When I came in the first time, they talked about it (DNR) before they started any treatment". The accompanying family member added to clarify: "Which was after having been diagnosed...you didn't know what the stage of cancer was at that time, but you were experiencing speech problems and had an emergency CT scan and they found complications with the brain" (brain metastasis). 


\section{Cureus}

\section{Trigger}

Admission to the community hospital

Brother brought it up

Radiation oncologist initiated the discussion

Pathology results

Patient himself initiated the discussion

Results of CT scan at a scheduled visit

Diagnosis of brain metastasis

Family physician clarified how patient wanted to be cared for
Patients (n)

1

1

1

1

2

1

2

1

\section{TABLE 5: Triggers to initiate DNR discussion}

Sub-theme: Who Should be Involved in the DNR Discussion?

Patients and CGs indicated their preference for the HCP and/or family member to be involved in DNR discussions. Most patients had their discussions about DNR with their physician. When specifically asked who else should be present during these discussions, most patients and their CGs preferred to have a family member or a support person present in addition to a physician. However, when patients and CGs were interviewed to describe their preference for the most appropriate HCP to initiate DNR discussions, the following categories of responses were identified: uncertainty and depends on the patient's situation.

The category of "uncertainty" captures statements by patients indicating their uncertainty about the appropriate HCP to discuss DNR. As one patient stated, "You know I probably didn't know who to put ... I don't know who would ... Dr. X (oncologist)? He's not my family physician." The second category, "depends on patient situation" reflects family members' reliance on the 'context' of the patient's condition to decide who is appropriate and 'when' to have the DNR conversation with the patient. As one CG stated, "Well, it would be the medical resident or a physician who felt that the condition warranted the discussion. You know, so it was trust in the medical system per se and I would presume in that emergency situation that discussion was with a resident, but I may be incorrect about that."

\section{Emotional response to DNR discussions}

The second main theme of 'Emotional response to DNR discussions' captures a qualitative account about the degree of distress that patients and CGs perceived they experienced (ranging from 'a little' to a 'moderate' amount on the investigator-developed emotional response survey item) when engaged in DNR discussions (Table 6). Most patients perceived that a DNR status would not compromise their care and felt that a DNR decision could be reversed. The following sub-themes reflect patients' emotional and cognitive acceptance of the situation. 


\section{Cureus}

\begin{tabular}{|l|l|l|}
\hline A little & Patients (n) & Caregivers (n) \\
\hline Moderate amount & 4 & 7 \\
\hline Not at all & 3 & 1 \\
\hline
\end{tabular}

TABLE 6: Emotional distress perceived by DNR discussions

Sub-theme: Acceptance of the Situation and Prognosis

The first sub-theme reflects patients' "Acceptance of the Situation and Prognosis" where one patient stated, "I just feel I've got it, what can I do ?" A "fighting spirit" reflected an attitude adopted by patients to deal with the disease and prognosis. One patient stated, "So I'm going to fight it. So there was no use being upset at that point; just crash on."

\section{Sub-theme: Definitely Distressing in an Emergent Situation}

The sub-theme of "Definitely Distressing in an Emergent Situation" reflects patients' and CGs' upset and anxiety when DNR is discussed in an emergency or unanticipated situation. One CG stated, "Yeah, I think that it's obviously distressing, the whole event that led to it was a distressing event in terms of entering Emergency with a prognosis that there were serious risks."

Sub-theme: Mixed Feelings

The sub-theme of "Mixed Feelings" reveals a mixture of hope tempered by an admission of imminent death by study participants. One patient stated, "I guess I'm sort of mixed feelings... I said we're going to beat it. So I'm still in that mind frame and I don't really know if I want to know whether I have a month or six months or a year."

Sub-theme: Nobody Wants to Talk About It

The sub-theme of "Nobody Wants to Talk About It" reflects statements by study participants indicating the taboo nature of talking about DNR. One patient shared, "I guess it's probably something nobody wants to talk about, but so you know I don't know how the reaction of how some people would react to it."

Sub-theme: Uncertain About His/Her Right to Change One's Mind About DNR Status

The sub-theme of "Uncertain About His/Her Right to Change One's Mind About DNR Status" across the illness trajectory reflects the participants' lack of understanding about being able to reverse one's decision about DNR status as time goes on. One patient explained, "Well, I don't know? If I am down the road and I'm that sick, I don't think I want to be revived ..."

Sub-theme: Expectations of Same Care

The last sub-theme of "Expectations of Same Care" reflects the participants' beliefs that 
regardless of the DNR status, the patient will continue to receive quality care. One patient shared, "I would like to get the very best of the same care that I've had, only in a different manner, you know. It would be a different ... I've had wonderful care."

\section{Suggestions to improve DNR discussions}

The third main theme, 'Suggestions to improve DNR discussions', reflects patient and CG recommendations on how to improve their comfort while discussing DNR and are reflected in five sub-themes.

Sub-theme: Pay Attention to Delivery

The first sub-theme, "Pay Attention to Delivery", speaks to the participants felt the need for a sensitive approach and assurance of attentive support during the DNR discussion. One patient stated, "I have found on occasion one of the doctors to be very blunt. I think there's different ways that you can come at it and I felt that this way has been not like Dr. X's". A caregiver added, "I don't know. I just think that make sure that those people are supportive to the individual if they're there are there and present."

Sub-theme: Respect and Awareness of Patient's Wishes

Sub-theme two, "Respect and Awareness of Patient's Wishes", reflects the wishes of participants for health care providers to engage in a patient-centered approach by paying attention to the belief, values, and desires of patients regarding DNR. One caregiver stated, "He [oncologist] understand what X [patient] would like to accomplish."

Sub-theme: Be Straightforward, Honest but Polite

The third sub-theme, "Be Straightforward, Honest but Polite", describes for honest and timely communication that is respectful toward the patient's need for clear-cut information. Different patients stated: "Be frank and blunt...Just be straight to people", "Start talking to people they are health, before they get sick", and "There's no way to whitewash it or do anything else but offer reassurance support."

Sub-theme: Expect the Physician to be Knowledgeable and Sensitive

The fourth sub-theme, "Expect the Physician to be Knowledgeable and Sensitive", explains participants' desire for sound medical knowledge that is shared in a caring manner while considering the patient's unique situation. One patient stated, "Be informed what kind of condition the patient is in."

Sub-theme: Understand the Physician's Viewpoint

The final sub-theme, "Understanding the Physician's Viewpoint", reflects the participants' desire for physicians to be transparent about their understanding of DNR. One caregiver explained, "I suppose one of the things that might have been discussed to seek clarification would be the physician's interpretation of what DNR means ... You know there is a grey area about just the scope of what DNR means."

\section{Discussion}

Optimizing the quality of life (QOL) of patients with incurable lung cancer requires attention to both physical symptoms (e.g., pain) and emotional distress regardless of the DNR status [4]. 
Our study is a reflection of how terminally ill lung cancer patients and their CGs feel and react when confronted with DNR discussions. In a meta-analysis, including 43 studies from 14 countries with terminal cancer patients, an enhanced sense of psycho-spiritual well-being was the key factor influencing the psychosocial well-being of the patients. Being aware of the prognosis and presence of family support contributed positively while emotional distress and hopelessness had an adverse effect on the emotional health of the patients [5].

Patients and CGs in our study desired a supportive health care environment and they preferred to have a family member present during the discussion. Emotional and physical supports from family members have profound effects on the QOL of dying patients [6]. The National Consensus Project for quality palliative care requires documentation of relationships and existing social networks as part of the social assessment by HCP for palliative care [7].

In our study, although some patient participants expressed uncertainty about the extent of their disease, they accepted the terminal nature of their cancer. It is difficult to explain this somewhat contradictory finding. However, terminally ill patients may differ from each other in the extent that they understand their disease extent and their prognosis $[5,8]$. In a Canadian study with 200 advanced cancer patients, those who did not acknowledge their prognosis were three times more likely to be depressed than those who had some acknowledgment of their prognosis [9]. Most patients desire to know their prognosis [1], which supports our study findings of participants' desire for honest and direct communication about their terminal state.

Most patients in our study held preconceived thoughts about their resuscitative status while other patients described acute situations that triggered DNR discussions. In a study with 1,081 colorectal and lung cancer patients, 55\% EOL care discussions occurred during an emergency room visit or a hospital admission [10].

Most of the current study's patients had DNR discussions with their oncologist or a family physician and usually in the presence of a family member. On the other hand, many patient participants expressed uncertainty when they stated, "depending on the situation" as to who they would identify as the appropriate HCP to initiate DNR discussions. In a Canadian study with 429 patients who visited their physician, $86 \%$ identified the family physician as their preferred HCP to discuss DNR, regardless of their DNR status [11]. Considering the uncertainty of our patients about their preferred HCP for DNR discussions, perhaps more collaboration is required between oncologists and the family physicians.

Both patients and CGs found DNR discussions somewhat distressing while maintaining hope and accepting the reality of being terminal. There were mixed feelings about the reversibility of a DNR decision and concerns about the care of patients after the DNR status had been decided. It is plausible that patients may acknowledge the terminal nature of the disease (cognitive acceptance) with or without accepting it at the emotional level. Cognitive and emotional acceptance of having an incurable and terminal cancer can have both a unique and a synergistic effect on EOL outcomes and are determined in part by how the prognosis was communicated by the HCP and how the patient perceives this communication [1]. Patients with only cognitive acceptance may be less peaceful and more symptomatic. On the contrary, emotional acceptance of the terminal nature of the disease is associated with feeling less terrified and more supported by others [12-13]. Furthermore, emotional 'acceptance' of the terminal nature of the disease is not a static event with the possibility that the patient may change his or her decision about the DNR status over time, which requires sensitivity understanding on the part of the health care team.

Both patients and their CGs desired their HCP to be emotionally sensitive, knowledgeable, and straightforward in his/her communication with a clear interpretation of DNR. This observation 
is in concordance with literature where patients described their desire for complete information, to be treated as a unique individual, and for trust in the competence of their physicians [14-18].

\section{Limitations}

Our pilot study sample size was too small for rigorous statistical analyses of quantitative responses. Themes and sub-themes reflected in this study are limited to the perceptions and preferences of patients and CGs who attended a single mid-prairie Province cancer care clinic located in an urban setting.

\section{Conclusions}

This cross-sectional pilot study uniquely presents in-depth analyses of thoughts and feelings held by patients with terminal lung cancer and their CGs in a tertiary academic center when engaged in DNR discussions. Observations and themes depicted in this study require further exploration in a larger and more comprehensive study. Regardless, we found that most patients and CGs were willing to talk openly about their experiences with psychosocial supports and emotional reactions that led to their recommendations for HCPs to improve DNR discussions in ways that support their unique desires, preferences, and values when dealing with terminal illness.

\section{Additional Information \\ Disclosures}

Human subjects: Consent was obtained by all participants in this study. Research Ethics Board University of Manitoba issued approval H2011:227. Animal subjects: All authors have confirmed that this study did not involve animal subjects or tissue. Conflicts of interest: In compliance with the ICMJE uniform disclosure form, all authors declare the following: Payment/services info: All authors have declared that no financial support was received from any organization for the submitted work. Financial relationships: All authors have declared that they have no financial relationships at present or within the previous three years with any organizations that might have an interest in the submitted work. Other relationships: All authors have declared that there are no other relationships or activities that could appear to have influenced the submitted work.

\section{Acknowledgements}

We sincerely thank Mrs. Kathy Suderman who, as the Administrative Head of the Department of Radiation Oncology CCMB, provided a generous financial support for this project.

\section{References}

1. Trice ED, Prigerson HG: Communication in end-stage cancer: review of the literature and future research. J Health Commun. 2009, 14:95-108. 10.1080/10810730902806786

2. Ahmed N, Lobchuk M, Hunter WM, Johnston P, Nugent Z, Sharma A, Ahmed S, Sisler J: How, when and where to discuss Do Not Resuscitate: A prospective study to compare the perceptions and preferences of patients, caregivers, and health care providers in a multidisciplinary lung cancer clinic. Cureus. 2015, 7:e257. 10.7759/cureus.257

3. Bradley EH, Curry LA, Devers KJ: Qualitative data analysis for health services research: developing taxonomy, themes, and theory. Health Serv Res. 2007, 42:1758-1772. 10.1111/j.1475-6773.2006.00684.x

4. Geddes DM: Quality of life in lung cancer . Respir Med. 1991, 85:7-11. 10.1016/S09546111(06)80162-9 
5. Lin HR, Bauer-Wu SM: Psycho-spiritual well-being in patients with advanced cancer: an integrative review of the literature. J Adv Nurs. 2003, 44:69-80. 10.1046/j.1365-

2648.2003.02768.x

6. Prince-Paul M: Understanding the meaning of social well-being at the end of life . Oncol Nurs Forum. 2008, 35:365-371. 10.1188/08.0NF.365-371

7. Colby WH, Dahlin C, Lantos J, Carney J, Christopher M: The National Consensus Project for Quality Palliative Care Clinical Practice Guidelines Domain 8: ethical and legal aspects of care. HEC Forum. 2010, 22:117-131. 10.1007/s10730-010-9128-3

8. Pronzato P, Bertelli G, Losardo P, Landucci M: What do advanced cancer patients know of their disease? A report from Italy. Support Care Cancer. 1994, 2:242-244. 10.1007/BF00365729

9. Chochinov HM1, Tataryn DJ, Wilson KG, Ennis M, Lander S: Prognostic awareness and the terminally ill. Psychosomatics. 2000, 41:500-504. 10.1176/appi.psy.41.6.500

10. Mack JW, Cronin A, Taback N, Huskamp HA, Keating NL, Malin JL, Earle CC, Weeks JC: Endof-life discussions among patients with advanced cancer: A cohort study. Ann Intern Med. 2012, 156:204-210. 10.1059/0003-4819-156-3-201202070-00008

11. Robinson C, Kolesar S, Boyko M, Berkowitz J, Calam B, Collins M: Awareness of do-notresuscitate orders: what do patients know and want?. Can Fam Physician. 2012, 58:e229-33.

12. Ray A, Block SD, Friedlander RJ, Zhang B, Maciejewski PK, Prigerson HG: Peaceful awareness in patients with advanced cancer. J Palliat Med. 2006, 9:1359-1368. 10.1089/jpm.2006.9.1359

13. Nilsson ME, Maciejewski PK, Zhang B, Wright AA, Trice ED, Muriel AC, Friedlander RJ, Fasciano KM, Block SD, Prigerson HG: Mental health, treatment preferences, advance care planning, location, and quality of death in advanced cancer patients with dependent children. Cancer. 2009, 115:399-409. 10.1002/cncr.24002

14. Arora NK: Interacting with cancer patients: the significance of physicians' communication behavior. Soc Sci Med. 2003, 57:791-806. 10.1016/S0277-9536(02)00449-5

15. Hagerty RG, Butow PN, Ellis PM, Dimitry S, Tattersall MH: Communicating prognosis in cancer care: a systematic review of the literature. Ann Oncol. 2005, 16:1005-1053.

10.1093/annonc/mdi211

16. Hagerty RG, Butow PN, Ellis PM, Lobb EA, Pendlebury SC, Leighl N, MacLeod C, Mac Leod C, Tattersall MH: Communicating with realism and hope: incurable cancer patients' views on the disclosure of prognosis. J Clin Oncol. 2005, 23:1278-1288. 10.1200/JCO.2005.11.138

17. Leydon GM, Boulton M, Moynihan C, Jones A, Mossman J, Boudioni M, McPherson K: Cancer patients' information needs and information seeking behaviour: in depth interview study. BMJ. 2000, 320:909-913. 10.1136/bmj.320.7239.909

18. Butow PN, Dowsett S, Hagerty R, Tattersall MH: Communicating prognosis to patients with metastatic disease: what do they really want to know? Support Care Cancer. 2002, 10:161168. 10.1007/s005200100290 